\title{
Rapid screening of the SARS-CoV-2 VOC 202012/01 B.1.1.7 variant using three different assays
}

\author{
Cristina Riazzo ${ }^{1}$, Rosa Pedraza-Merino ${ }^{1}$, Laura Merino ${ }^{2}$, Pedro Camacho ${ }^{2}$, Jose A \\ Lepe-Jimenez $^{2}$, Luis Martinez-Martinez ${ }^{1}$, and Manuel Causse-del Rio ${ }^{1}$ \\ ${ }^{1}$ Reina Sofia University Hospital \\ ${ }^{2}$ Virgen del Rocio University Hospital
}

September 24, 2021

\begin{abstract}
Background The aim of this study was to evaluate three commercially available methods (Allplex SARS-CoV-2 Assay, Allplex SARS-CoV-2/FluA/FluB/RSV Assay and Novaplex SARS-CoV-2 Variants I Assay) for screening of the SARS-CoV-2 VOC 202012/01 B.1.1.7 variant. Methods A total of 160 nasopharyngeal samples (150 positive and 10 negative for SARS-CoV-2) were tested with all three molecular assays. Next-generation sequencing (NGS) was used as the reference method to determine analytical performance. Results Total (100\%) agreement was found for SARS-CoV-2 detection with all three assays. For B.1.1.7 screening, the sensitivity of the Allplex SARS-CoV-2 Assay, the Allplex SARS-CoV-2/FluA/FluB/RSV Assay and the Novaplex SARS-CoV-2 Variants I Assay (Seegene Inc.) were $94.5 \%, 98.7 \%$ and $100 \%$, respectively, while the specificities of the assays were $98.6 \%, 81.7 \%$ and $100 \%$, respectively. Conclusions Although the best results for identifying the B.1.1.7 variant in this study were achieved with the Novaplex Variants I Assay, the three approaches evaluated can be considered cost-effective primary screening tools to rapidly monitor the VOC 202012/01 B.1.1.7 variant.
\end{abstract}

\section{Hosted file}

Manuscript.docx available at https://authorea.com/users/435560/articles/538496-rapidscreening-of-the-sars-cov-2-voc-202012-01-b-1-1-7-variant-using-three-different-assays 